\title{
The pathobiology of Mycobacterium abscessus revealed through phenogenomic analysis
}

Lucas Boeck ${ }^{1,2,3,4}$, Sophie Burbaud ${ }^{1,2}$, Marcin Skwark ${ }^{5}$, Will H. Pearson ${ }^{6,7}$, Jasper Sangen ${ }^{1,2}$, Aaron Weimann ${ }^{1,2}$, Isobel Everall ${ }^{3}$, Josephine $M$ Bryant $^{1,2}$, Sony Malhotra ${ }^{5,8}$, Bridget $P$. Bannerman $^{1,2,5}$, Katrin Kierdorf ${ }^{6,7,9}$, Tom L. Blundell ${ }^{5}$, Marc S. Dionne ${ }^{6,7}$, Julian Parkhill ${ }^{10}, \mathrm{R}$. Andres Floto ${ }^{1,2,11}$

1. Molecular Immunity Unit, University of Cambridge Department of Medicine, MRC Laboratory of Molecular Biology, Cambridge, UK

2. Cambridge Centre for $\mathrm{Al}$ in Medicine, Cambridge, UK

3. Wellcome Sanger Institute, Hinxton, UK

4. Department of Biomedicine, University of Basel, Basel, Switzerland

5. Department of Biochemistry, University of Cambridge, Tennis Court Road, Cambridge, UK

6. MRC Centre for Molecular Bacteriology and Infection, Imperial College London, London, UK

7. Department of Life Sciences, Imperial College London, London, UK

8. Scientific Computing Department, Science and Technology Facilities Council, Harwell, UK

9. Institute of Neuropathology, Faculty of Medicine, University of Freiburg, Freiburg, Germany

10. Department of Veterinary Medicine, University of Cambridge, Cambridge, UK

11. Cambridge Centre for Lung Infection, Royal Papworth Hospital, Cambridge, UK

Correspondence to:

R. Andres Floto

Arf27@cam.ac.uk 


\section{ABSTRACT}

The medical and scientific response to emerging pathogens is often severely hampered by ignorance of the genetic determinants of virulence, drug resistance, and clinical outcomes that could be used to identify therapeutic drug targets and forecast patient trajectories ${ }^{1-5}$. Taking the newly emergent multidrug-resistant bacteria Mycobacterium abscessus as an example ${ }^{6}$, we show that combining high dimensional phenotyping with whole genome sequencing in a phenogenomic analysis can rapidly reveal actionable systems-level insights into bacterial pathobiology. Using in vitro and in vivo phenotyping, we discovered three distinct clusters of isolates, each associated with a different clinical outcome. We combined genome-wide association studies (GWAS) with proteome-wide computational structural modelling ${ }^{7}$ to define likely causal variants, and employed direct coupling analysis (DCA) ${ }^{8}$ to identify co-evolving, and therefore potentially epistatic, gene networks. We then used in vivo CRISPR-based silencing to validate our findings, defining a novel secretion system controlling virulence in $M$. abscessus, and illustrating how phenogenomics can reveal critical pathways within emerging pathogenic bacteria.

\section{INTRODUCTION}

Over the last two decades, $M$. abscessus, a rapid growing species of nontuberculous mycobacteria (NTM), has emerged as a major threat to individuals with Cystic Fibrosis (CF) and other chronic lung disease ${ }^{9}$. Rates of infection of CF patients have increased around the World ${ }^{9-11}$, in part due to hospital-based person-to-person transmission ${ }^{12,13}$ and the emergence of globally-spread dominant circulating clones that are associated with increased virulence and worse clinical outcomes ${ }^{14}$. Infections with $M$. abscessus are challenging and sometimes impossible to treat ${ }^{9,15,16}$, lead to accelerated inflammatory lung damage ${ }^{17,18}$, and may prevent safe transplantation ${ }^{19}$.

To date, very little is known about how $M$. abscessus infects humans ${ }^{6}$, how it causes inflammatory lung damage, and how it resists antibiotics ${ }^{6}$. There is thus an urgent need to better understand the pathophysiology of $M$. abscessus, to define optimal drug targets, and to predict the virulence and antibiotic susceptibility of clinical isolates.

We therefore sought to combine detailed in vitro and in vivo phenotyping, whole genome sequencing, computational structural modelling, and epistatic analysis to provide a phenogenomic map of $M$. abscessus that might define critical pathways involved in virulence and drug resistance. 


\section{RESULTS}

We first characterised 331 clinical $M$. abscessus isolates across 58 phenotypic dimensions exploring five key pathogenic traits: planktonic growth in different carbon sources; antibiotic resistance (at early and late time points) against a selection of drugs recommended by clinical treatment guidelines ${ }^{9}$; in vitro infection of a human macrophage cell line model (differentiated THP1 cells), monitored using high content confocal microscopy; in vivo infection of Drosophila melanogaster, measuring host survival and inflammatory responses; and clinical outcomes following infection, available through previously collected metadata ${ }^{14}$ (Figure 1a,

\section{Supplementary Figure 1).}

We examined the relationship between phenotypes, finding correlations within, and sometimes between, pathogenic traits (Figure 1b, Supplementary Figure 2). To explore whether there were distinct patterns of bacterial behaviours, we used experimentally-derived data to plot individual isolates in phenotypic space, identifying three discrete groups, each associated with different clinical outcomes (Figure 1c,d, Supplementary Figure 3), representing distinct heritable traits (Supplementary Figure 4). Isolates in Groups 1, 2, and 3 demonstrated progressively faster growth in liquid culture and within macrophages, higher rates of macrophage and Drosophila death, and greater inflammatory responses. While Group 2 isolates were associated with the most favourable clinical outcome, potentially related to their macrolide susceptibility (a key determinant of treatment response ${ }^{20,21}$ ), we found that Group 1 and Group 3 isolates, although similarly macrolide resistant, had very different clinical outcomes, highlighting the importance of other phenotypic characteristics in determining prognosis, and suggesting that more virulent (and thus immunogenic) isolates might be cleared more easily by patients (as previously reported for other pathogenic bacteria ${ }^{22-25}$ ).

To understand the genetic basis for these important variations in $M$. abscessus behaviour, we used whole genome sequence data to perform a genome-wide association study (GWAS) for each phenotype (Figure 2a), evaluating approximately 270,000 genetic variants comprising single nucleotide polymorphisms (SNPs), insertions, and deletions. We used mixed models corrected for population structure ${ }^{26}$ to identify locus effects, as well as uncorrected linear models to ensure we captured lineage effects ${ }^{27}$. In total, we identified 1926 hits (involving 1000 genes) across 46 phenotypes (Supplementary Table 1). These included previously known genetic determinants, such as the $16 \mathrm{~S}$ and $23 \mathrm{~S}$ rRNA mutations associated with constitutive aminoglycoside and macrolide resistance $\left(p=1.3 \times 10^{-75}\right.$ and $p=1.5 \times 10^{-54}$ respectively; Supplementary Figure 5), thereby confirming the effectiveness of our approach.

Current GWAS approaches are limited in their ability to accurately identify causal variants by both the presence of linkage disequilibrium, which in the case of $M$. abscessus (as with other 
bacteria ${ }^{28,29}$ ) is extensive and genome-wide (Figure 2a, Supplementary Figure 6), and by a failure to consider the impact of mutations on protein function ${ }^{30,31}$.

We therefore applied proteome-wide computational structural modelling to evaluate the likely functional impact of all nonsynonymous SNPs across the genome, by applying our graphbased machine learning method $\mathrm{mCSM}^{7}$ to our comprehensive $M$. abscessus structural database Mabellini ${ }^{32}$ (Figure $\mathbf{2 b}$ ), in order to identify likely causal mutations.

As an example, the GWAS for intracellular replication of $M$. abscessus within macrophages identified a number of hits at genome-wide significance including a cluster of variants within the mycobactin operon (Figure 2c), containing genes involved in iron scavenging ${ }^{33,34}$. Structural modelling predicted that one variant, a missense mutation (Ile256Thr) in the mycobactin polyketide synthetase $(m b t D)$ gene, was most likely to result in loss of protein function and therefore be causally related to the phenotypic change, probably through altering the ability of intracellular M. abscessus to access iron.

To understand whether mutations across the genome might have co-evolved, indicating potential epistatic interactions between genes, we deployed correlation-compressed direct coupling analysis $\left(\mathrm{ccDCA}^{8}\right)$ on whole genome sequences from 2366 clinical isolates of $M$. abscessus to identify whether variant co-occurrence deviated from the expected frequencies based on linkage disequilibrium ${ }^{35,36}$, and thus indicate evolutionary co-selection. We evaluated $10^{12}$ potential couplings (resulting from approximately $10^{6}$ genetic variants), and identified 1,168,913 that were significantly enriched (accepting a false discovery rate of $10^{-6}$; Figure 3a, Supplementary Figure 7). We found many enriched couplings between known or predicted virulence genes (Figure 3b, Supplementary Table 2), indicating pathogenic evolution of $M$. abscessus (as previous identified ${ }^{14,37}$ ). We used the ranked outputs from the ccDCA analysis to establish discrete networks of genes that have co-evolved, and thus probably functionally interact (Figure 3c). As examples, we find highly connected clusters of: mammalian cell entry (MCE) genes (Figure 3d), implicated in controlling adhesion, uptake, and intracellular survival within macrophages ${ }^{38,39}$; mycobactin synthesis genes (Figure 3e), including some identified through GWAS analysis (Figure 2c,d); and genes involved in bacterial secretion systems.

Finally, we sought to integrate outputs from our detailed multidimensional phenotyping, structure-guided GWAS analysis, and DCA-based epistatic mapping, to achieve a systemslevel understanding of the genetic basis for important pathological processes in M. abscessus. We focused on in vivo infection in Drosophila, a model that replicates many of the features of human mycobacterial infection (Figure 4a) ${ }^{40-43}$. Amongst the top hits from our structureguided GWAS analysis (Figure 4b, Supplementary Figure 8) were a deletion in a component 
of a putative Type II secretion system (MAB_0471) and a deleterious mutation in a nonribosomal peptide synthetase (MAB_3317c). Both variants had independently arisen as homoplastic mutations across the $M$. abscessus phylogenetic tree (Figure 4c), including within the ancestor of one of the dominant circulating clones (DCC2) of M. a. abscessus, responsible for several transmission networks amongst CF patients ${ }^{13,14}$. We found that isolates with deletions in the Type II secretion system were associated with prolonged survival of infected Drosophila and more persistent clinical infection of CF patients (Figure $4 \boldsymbol{d}$ ).

We sought to experimentally validate both these GWAS hits through inducible CRISPR-based transcriptional silencing (CRISPRi) as previously described ${ }^{44}$. Although we found no effect of gene silencing on growth in liquid media, silencing of either MAB_0471 or MAB_3317c during in vivo infection significantly increased Drosophila survival (Figure 4e, Supplementary Figure 9), indicating that these genes regulate $M$. abscessus virulence.

Our DCA analysis revealed that both these GWAS hits were part of a discrete network of probably epistatic genes involved in bacterial secretion, cell wall biosynthesis, metabolism, and transcriptional regulation (Figure 4f, Supplementary Figure 10). To experimentally test this prediction of epistasis, we selected another gene from the same network (MAB_0472) and transcriptionally silenced it during in vivo infection. We found that Drosophila survival was also increased by its CRISPRi knockdown (Figure $\mathbf{4 g}$ ), suggesting that all three genes are functionally interacting.

Thus, phenogenomic analysis can accurately identify critical gene networks responsible for virulence and other characteristics in poorly understood bacterial pathogens such as $M$. abscessus. Our approach of integrating computational structural modelling with conventional GWAS analyses and DCA-driven mapping of gene interaction networks has revealed key determinants of $M$. abscessus antibiotic resistance and virulence.

Importantly we have identified a novel and important gene network, involving a previously unidentified peptide synthetase and type 2 secretion system regulating, that regulates the pathogenic potential of $M$. abscessus and could be targeted therapeutically. Phenogenomic analysis should be readily applicable to any pathogen, permitting rapid identification of prognostic indicators and novel potential drug targets. 


\section{FIGURE LEGEND}

\section{Figure 1: Multidimensional phenotyping of $M$. abscessus}

(a) Phenotypic variability of clinical $M$. abscessus isolates was assessed across multiple dimensions (described in Supplementary Methods) including: (i) Planktonic growth (assessed by serial OD measurement) in a range of different carbon sources; (ii) Minimal inhibitory concentrations (MIC) of a range of clinically relevant antibiotics were assessed on day 3 (MIC early) and day 11 (MIC late) to quantify intrinsic and inducible drug resistance; (iii) Macrophage infection (4h post infection), intracellular replication ( $2 \mathrm{~d}$ post infection) and death (2d post infection) were quantified using high-content imaging of differentiated THP-1 cells incubated with tdTomato-expressing clinical isolates; (iv) Survival and immune response of Drosophila melanogaster infected with clinical isolates; and (v) Clinical outcomes (lung function decline and clearance of $M$. abscessus from sputum samples) of infected patients (b) Pearson correlations within and across phenotypic groups shown as a matrix, with non-significant ( $p>$ 0.05 ) associations shown in white. (c) Clustering of clinical isolates, using k-means and Tstochastic neighbour embedding, based on (i) experimentally observed phenotypes only, demonstrate three distinct groups that (ii) differ in their clinical outcomes. (d) Distribution of specific phenotypes across the three phenotypic groups. $\mathrm{P}$ values calculated using Chisquared test or one-way analysis of variance, as appropriate.

\section{Figure 2: Integrating computational structural modelling into genome-wide association studies.}

(a) Genome-wide associations were performed for all phenotypes with the top variants extracted (up to 5 per association) and ordered using hierarchical clustering (red linear model; blue mixed model). Pairwise $r^{2}$ measurements of the identified genetic variants (grey scale) show extensive genome-wide linkage (LD). (b) To identify causal variants and overcome LD, the functional impacts of genetic variants were classified as having high effects (large deletions, frameshifts, start/stop alterations; red); moderate effects (inframe insertions/deletions; blue and green); and low effects (synonymous and intergenic variants; grey). The impact of missense mutations were estimated using proteome-wide computational structural modelling with variants considered as having high ( $r e d)$, moderate (blue) or low (green) functional effects based on terciles of the change in protein stability, estimated using mCSM. (c) Manhattan plot of the mixed model GWAS analysis of 264,122 genetic variants for intracellular $M$. abscessus replication. Several loci in $m b t D$, including 4 missense mutations, were identified as potential mechanisms relevant for intracellular $M$. abscessus survival. Inset: Protein model of MbtD with the high effect missense mutation lle256Thr shown in red. 


\section{Figure 3: Analysis of genome-wide epistasis through mutational co-evolution.}

Direct coupling analysis (ccDCA) was used to identify coevolving variants among $\sim 10^{12}$ potential variant combinations of 2,366 clinical $M$. abscessus isolates. (a) Circos plot of the $M$. abscessus chromosome showing the 100,000 top variant-to-variant couplings with a distance of $>100 \mathrm{bp}$ (black lines), coupling density (green; range 0-56307 couplings per 5kb) and SNP density (red; range 14-1961 SNPs per 5kb). (b) Significant variant-variant couplings identified through DCA were pooled as gene-gene couplings and ranked by the number of couplings. (c) Networks of co-evolving (and therefore likely epistatic) genes, based on DCA derived genegene couplings, colour coded by functional class. The strength and number of couplings shown by edge colour and thickness respectively. (d, e) Examples of highly coupled gene networks, highlighted by circles in (c), involving (d) mammalian cell entry proteins, and (e) components of the mycobactin biosynthesis pathway.

Figure 4: Defining genetic determinants of in vivo virulence in $M$. abscessus.

(a) Drosophila melanogaster infected with $M$. abscessus (magenta) resembles mycobacterial infection in other organisms; with infection of phagocytes (green) and formation of granulomalike structures (inset). (b) Genome-wide association (using a linear model) reveals a putative secretion-system protein and a peptide synthetase to be highly associated with Drosophila survival. (c) Both variants align to clinical isolates with long survival, including a dominant circulating clone, within the subspecies M. a. abscessus. (d) Deletion in MAB_0471 was associated with persistent respiratory infection in cystic fibrosis patients. (e) CRISPR/dCas9 knockdown of MAB_0471 and MAB_3317 (unlike the essential gene yidC) did not affect growth in liquid culture (left panel) but in vivo silencing did lead to prolonged survival of infected Drosophila, as shown by Kaplan-Meier survival curves generated from data from at least 18 infected flies per bacterial strain. (f) Epistatic gene network, derived from DCA outputs, revealed direct coupling of $M A B \_0471$ with other putative secretion system proteins including MAB_0472 and a distant connection to the peptide synthetase MAB_3317. (g) In vivo silencing of MAB_0472 replicated virulence attenuation. 


\section{Acknowledgements}

We would like to thank John Lees, Philip H. C. Kremer and Simon Harris for statistical and bioinformatical support.

Funding: This work was supported by The Wellcome Trust (107032AIA (RAF , SB), 10224/Z/15/Z JMB, 098051(JP)); The UK Cystic Fibrosis Trust (Innovation Hub grant 001 (RAF, TLB, JP, SB), SRC 002 \& 010 (TLB, JP, RAF); a Vertex Innovation award (RAF); NIHR Cambridge Biomedical Research Centre (RAF); and The Botnar Foundation (6063; RAF, AW, TLB, SM, JP). LB was supported by the Swiss National Science Foundation (P300PB_161024, P3P3PB_177799, PZ00P3_185792) and the Bangerter-Rhyner Foundation. LB is the recipient of a joint ERS/EMBO Long-Term Research fellowship $n^{\circ}$ LTRF 2015-5825. KK was supported by a DFG fellowship.

Author contributions: LB and RAF conceived the project and wrote the manuscript. LB, SB and JS performed the in vitro experiments. LB, WHP, KK and MSD performed the in vivo experiments. MS and SM performed the computational structural modelling. LB and MS performed direct coupling analysis. LB, AW, IE, JB and BB performed other bioinformatic analyses. SB developed the $M$. abscessus CRISPR interference technique. LB, SB and JS generated bacterial knockdown strains. TLB, MSD, JP and RAF provided supervisory support.

Competing interests: none

Ethics approval: Ethical approval was obtained from the National Research Ethics

Service (NRES; REC reference: 12/EE/0158) and the National Information Governance Board (NIGB; ECC 3-03 (f)/2012) for centres in England and Wales; from NHS Scotland Multiple Board Caldicott Guardian Approval (NHS Tayside AR/SW) for Scottish centres; and locally for other centres.

Data availability: All sequencing data of this study is deposited in the European Nucleotide Archive 


\section{REFERENCES}

1. Coll, F. et al. Genome-wide analysis of multi- and extensively drug-resistant Mycobacterium tuberculosis. Nature genetics 50, 307-316 (2018).

2. Miotto, O. et al. Genetic architecture of artemisinin-resistant Plasmodium falciparum. Nat Genet 47, 226-234 (2015).

3. Fraser, C. et al. Virulence and Pathogenesis of HIV-1 Infection: An Evolutionary Perspective. Science 343, 1243727 (2014).

4. Laabei, M. et al. Predicting the virulence of MRSA from its genome sequence. Genome Res 24, 839-849 (2014).

5. Walker, T. M. et al. Whole-genome sequencing for prediction of Mycobacterium tuberculosis drug susceptibility and resistance: a retrospective cohort study. Lancet Infect Dis 15, 1193-1202 (2015).

6. Johansen, M. D., Herrmann, J.-L. \& Kremer, L. Non-tuberculous mycobacteria and the rise of Mycobacterium abscessus. Nature reviews. Microbiology 18, 392-407 (2020).

7. Pires, D. E. V., Ascher, D. B. \& Blundell, T. L. mCSM: predicting the effects of mutations in proteins using graph-based signatures. Bioinformatics 30, 335-342 (2014).

8. Gao, C.-Y., Zhou, H.-J. \& Aurell, E. Correlation-compressed direct-coupling analysis. Physical Review E 98, 032407 (2018).

9. Floto, R. A. et al. US Cystic Fibrosis Foundation and European Cystic Fibrosis Society consensus recommendations for the management of non-tuberculous mycobacteria in individuals with cystic fibrosis. in vol. 71 Suppl 1 il-22 (2016).

10. Richards, C. \& Olivier, K. Nontuberculous Mycobacteria in Cystic Fibrosis. Semin Resp Crit Care 40, 737-750 (2019).

11. Martiniano, S. L., Nick, J. A. \& Daley, C. L. Nontuberculous Mycobacterial Infections in Cystic Fibrosis. Thorac Surg Clin 29, 95-108 (2019).

12. Renna, M. et al. Azithromycin blocks autophagy and may predispose cystic fibrosis patients to mycobacterial infection. Journal of Clinical Investigation 121, 3554-3563 (2011).

13. Bryant, J. M. et al. Whole-genome sequencing to identify transmission of Mycobacterium abscessus between patients with cystic fibrosis: a retrospective cohort study. Lancet $\mathbf{3 8 1}$, $1551-1560$ (2013).

14. Bryant, J. M. et al. Emergence and spread of a human-transmissible multidrug-resistant nontuberculous mycobacterium. Science 354, 751-757 (2016). 
15. Daley, C. L. et al. Treatment of Nontuberculous Mycobacterial Pulmonary Disease: An Official ATS/ERS/ESCMID/IDSA Clinical Practice Guideline. Clin Infect Dis 71, 905-913 (2020).

16. Jhun, B. W. et al. Prognostic factors associated with long-term mortality in 1445 patients with nontuberculous mycobacterial pulmonary disease: a 15-year follow-up study. The European respiratory journal 55, 1900798 (2020).

17. Esther, C. R., Esserman, D. A., Gilligan, P., Kerr, A. \& Noone, P. G. Chronic Mycobacterium abscessus infection and lung function decline in cystic fibrosis. J Cyst Fibros 9, 117-123 (2010).

18. Qvist, T. et al. Comparing the harmful effects of nontuberculous mycobacteria and Gram negative bacteria on lung function in patients with cystic fibrosis. J Cyst Fibros 15, 380-385 (2016).

19. Kavaliunaite, E. et al. Outcome according to subspecies following lung transplantation in cystic fibrosis pediatric patients infected with Mycobacterium abscessus. Transpl Infect Dis 22, e13274 (2020).

20. Choi, H. et al. Clinical Characteristics and Treatment Outcomes of Patients with Acquired Macrolide-Resistant Mycobacterium abscessus Lung Disease. Antimicrob Agents Ch 61, e01146-17 (2017).

21. Choi, G.-E. et al. Macrolide Treatment for Mycobacterium abscessus and Mycobacterium massiliense Infection and Inducible Resistance. Am J Resp Crit Care 186, 917-925 (2012).

22. Broder, U. N., Jaeger, T. \& Jenal, U. LadS is a calcium-responsive kinase that induces acute-to-chronic virulence switch in Pseudomonas aeruginosa. Nature microbiology 2, 16184 (2016).

23. Avican, K. et al. Reprogramming of Yersinia from Virulent to Persistent Mode Revealed by Complex In Vivo RNA-seq Analysis. Plos Pathog 11, e1004600 (2015).

24. Ronin, I., Katsowich, N., Rosenshine, I. \& Balaban, N. Q. A long-term epigenetic memory switch controls bacterial virulence bimodality. Elife 6, e19599 (2017).

25. Ernst, C. M. et al. Adaptive evolution of virulence and persistence in carbapenemresistant Klebsiella pneumoniae. Nat Med 26, 705-711 (2020).

26. Zhou, X. \& Stephens, M. Genome-wide efficient mixed-model analysis for association studies. Nature genetics 44, 821-824 (2012).

27. Earle, S. G. et al. Identifying lineage effects when controlling for population structure improves power in bacterial association studies. Nature microbiology 1, (2016).

28. Chen, P. E. \& Shapiro, B. J. The advent of genome-wide association studies for bacteria. Current opinion in microbiology 25, 17-24 (2015). 
29. Feil, E. J. \& Spratt, B. G. Recombination and the population structures of bacterial pathogens. Annu Rev Microbiol 55, 561-590 (2001).

30. Boucher, J. I., Bolon, D. N. A. \& Tawfik, D. S. Quantifying and understanding the fitness effects of protein mutations: Laboratory versus nature. Protein Sci 28, 671-671 (2019).

31. Gasperini, M., Starita, L. \& Shendure, J. The power of multiplexed functional analysis of genetic variants. Nat Protoc 11, 1782-1787 (2016).

32. Skwark, M. J. et al. Mabellini: a genome-wide database for understanding the structural proteome and evaluating prospective antimicrobial targets of the emerging pathogen Mycobacterium abscessus. Database : the journal of biological databases and curation 2019, 4471 (2019).

33. Voss, J. J. D. et al. The salicylate-derived mycobactin siderophores of Mycobacterium tuberculosis are essential for growth in macrophages. Proceedings of the National Academy of Sciences 97, 1252-1257 (2000).

34. Luo, M., Fadeev, E. A. \& Groves, J. T. Mycobactin-mediated iron acquisition within macrophages. Nat Chem Biol 1, 149-153 (2005).

35. Skwark, M. J. et al. Interacting networks of resistance, virulence and core machinery genes identified by genome-wide epistasis analysis. PLoS genetics 13, e1006508 (2017).

36. Puranen, S. et al. SuperDCA for genome-wide epistasis analysis. Microbial genomics 4, 310 (2018).

37. Bryant, J. M. et al. Stepwise pathogenic evolution of Mycobacterium abscessus. Science 372, eabb8699 (2021).

38. Chitale, S. et al. Recombinant Mycobacterium tuberculosis protein associated with mammalian cell entry. Cell Microbiol 3, 247-254 (2001).

39. Rengarajan, J., Bloom, B. R. \& Rubin, E. J. Genome-wide requirements for Mycobacterium tuberculosis adaptation and survival in macrophages. P Natl Acad Sci Usa 102, 8327-8332 (2005).

40. Dionne, M. S., Ghori, N. \& Schneider, D. S. Drosophila melanogaster is a genetically tractable model host for Mycobacterium marinum. Infection and immunity 71, 3540-3550 (2003).

41. Pean, C. B. et al. Regulation of phagocyte triglyceride by a STAT-ATG2 pathway controls mycobacterial infection. Nature Communications 8, 1-11 (2017).

42. Oh, C.-T., Moon, C., Jeong, M. S., Kwon, S.-H. \& Jang, J. Drosophila melanogaster model for Mycobacterium abscessus infection. Microbes Infect 15, 788-795 (2013).

43. Oh, C.-T., Moon, C., Park, O. K., Kwon, S.-H. \& Jang, J. Novel drug combination for Mycobacterium abscessus disease therapy identified in a Drosophila infection model. $J$ Antimicrob Chemoth 69, 1599-1607 (2014). 
bioRxiv preprint doi: https://doi.org/10.1101/2021.10.18.464689; this version posted October 18,2021 . The copyright holder for this

preprint (which was not certified by peer review) is the author/funder, who has granted bioRxiv a license to display the preprint in perpetuity. It is made available under aCC-BY 4.0 International license.

44. Rock, J. M. et al. Programmable transcriptional repression in mycobacteria using an orthogonal CRISPR interference platform. Nature microbiology 2, 16274 (2017). 
Firg biqRiv preprint doi: https://doi.org/10.1101/2021.10.18.464689; this version posted October 18, 2021. The copyright holder for this FIgure preprint (which was not certified by peer review) is the author/funder, who has granted bioRxiv a license to display the preprint in a. perpetuity. It is made available under aCC-BY 4.0 International license.

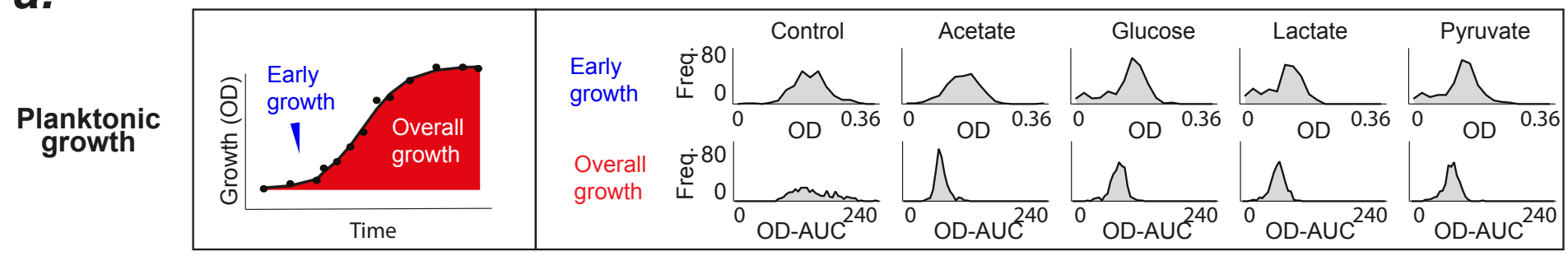

\section{Antibiotic resistance}

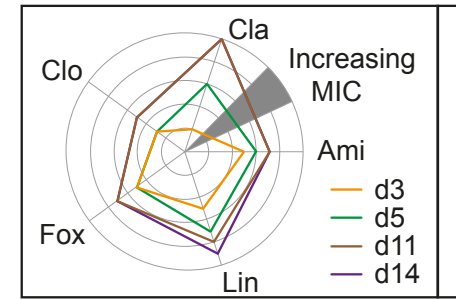

$\square$ MIC early $\square$ MIC late
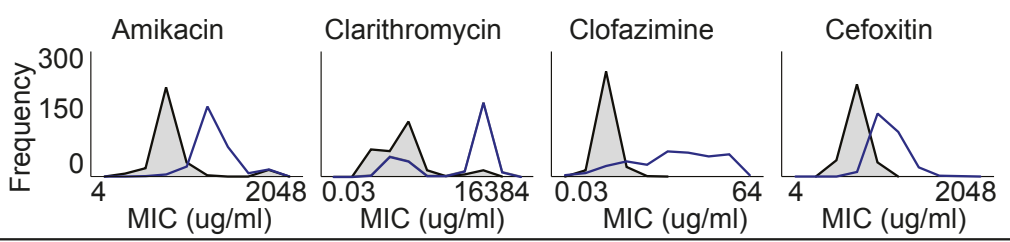

Linezolid
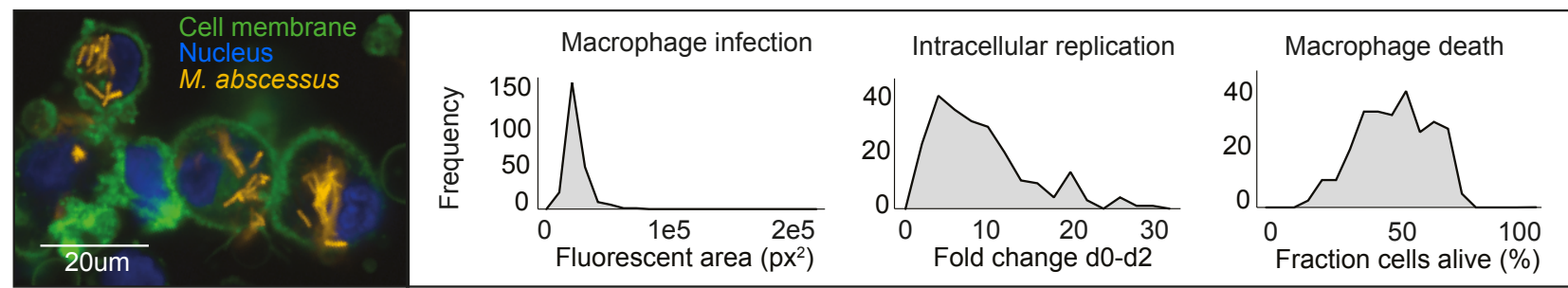

\section{In vivo \\ infection}

- Mab infected

- Uninfected

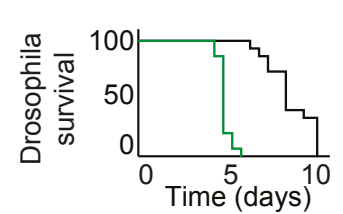

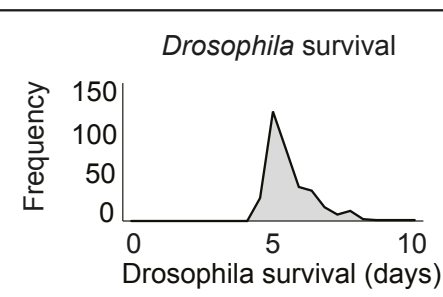
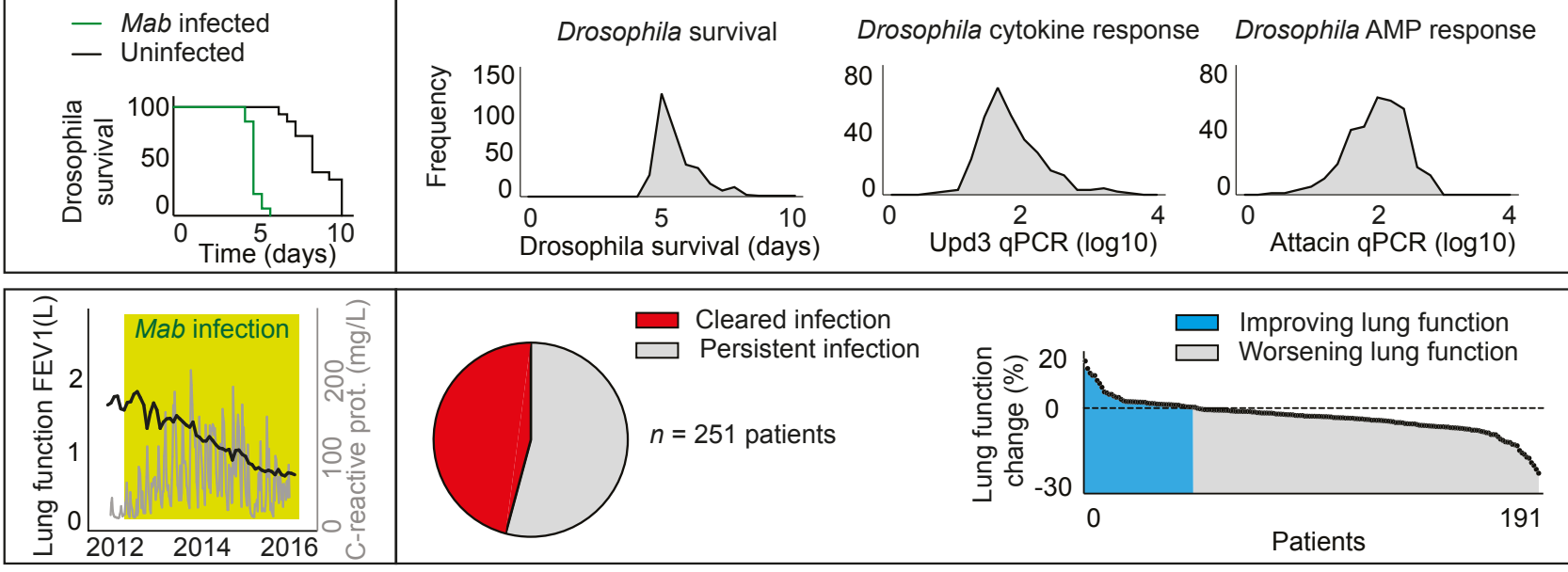

Clinical

outcome

b.

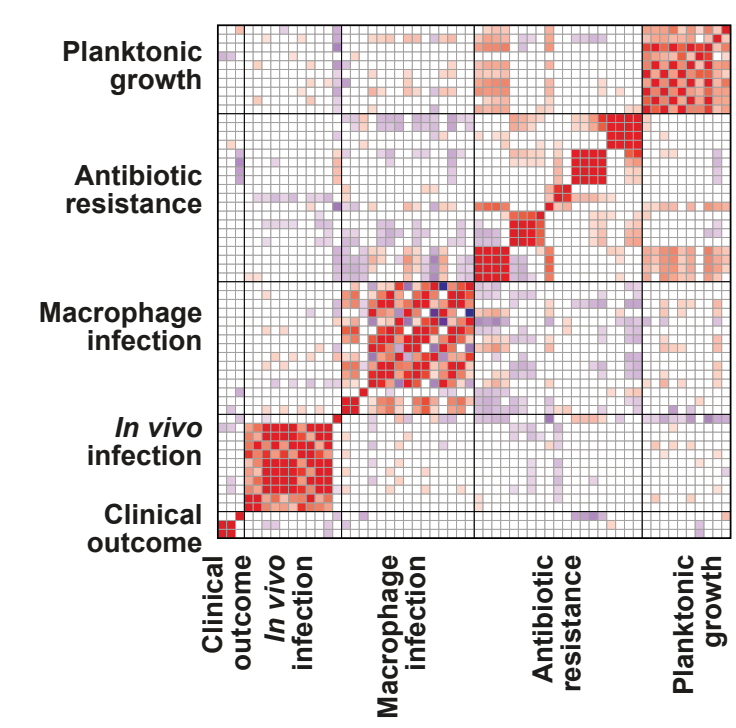

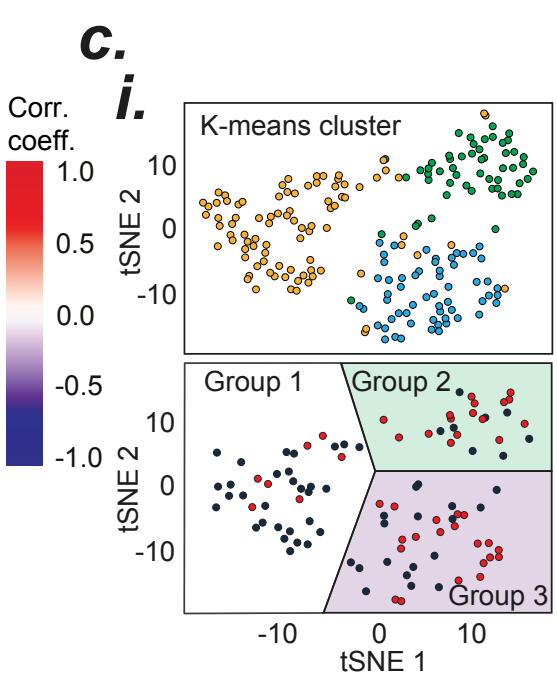

ii. infection: $\square$ Persistent d.

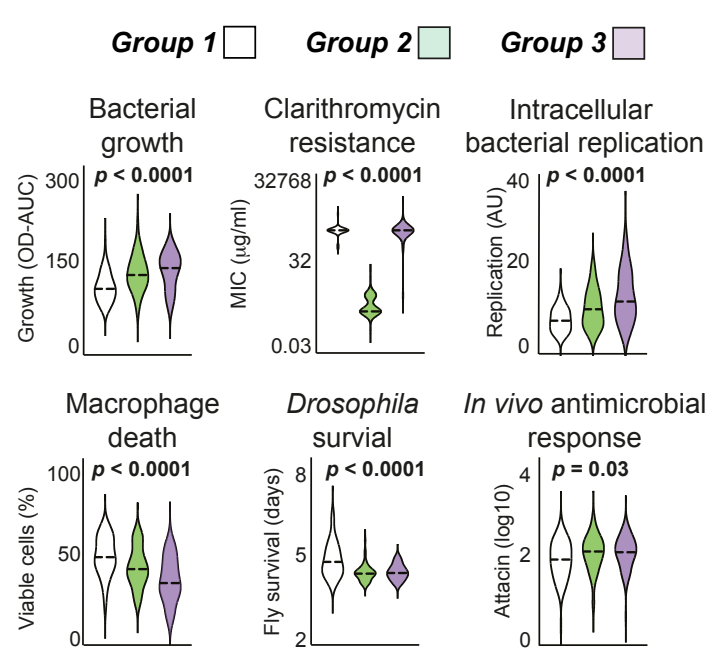

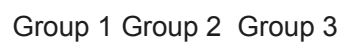

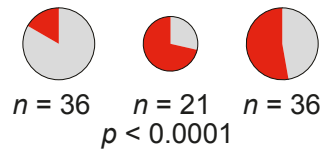




\section{Figure 2}

a.

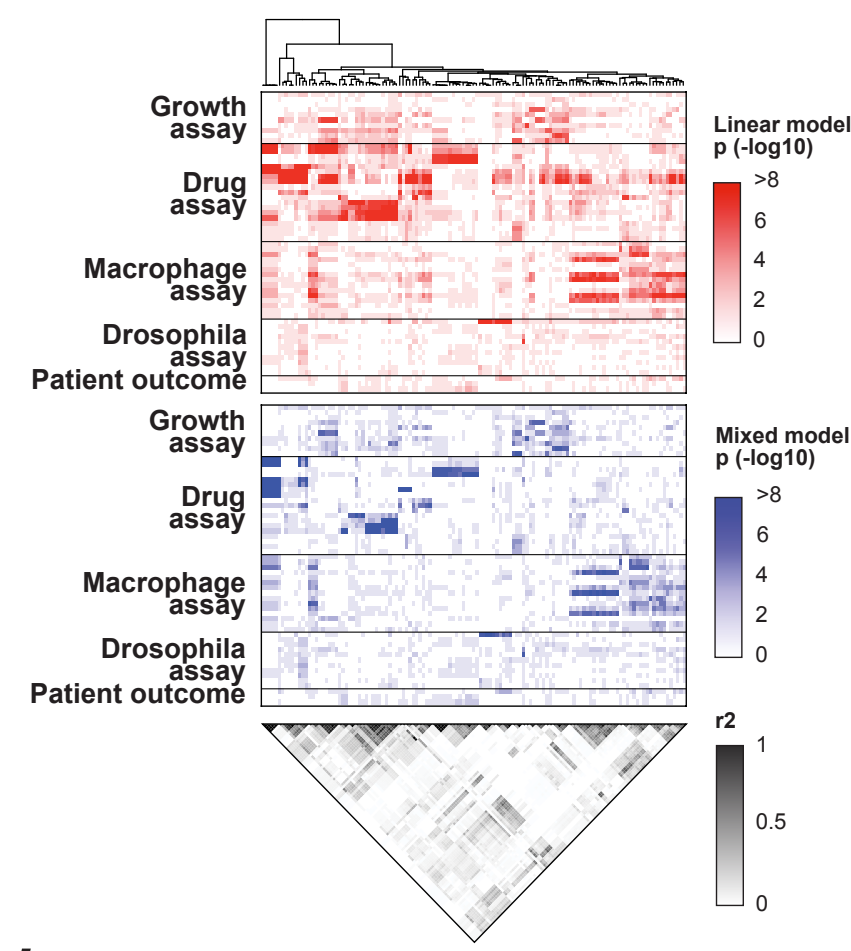

b.

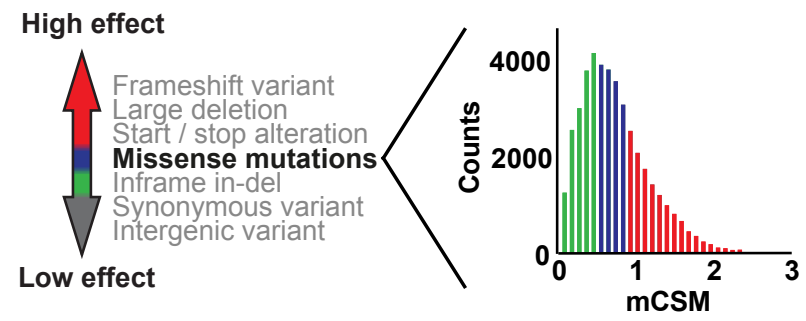

c. Intracellular M. abscessus replication

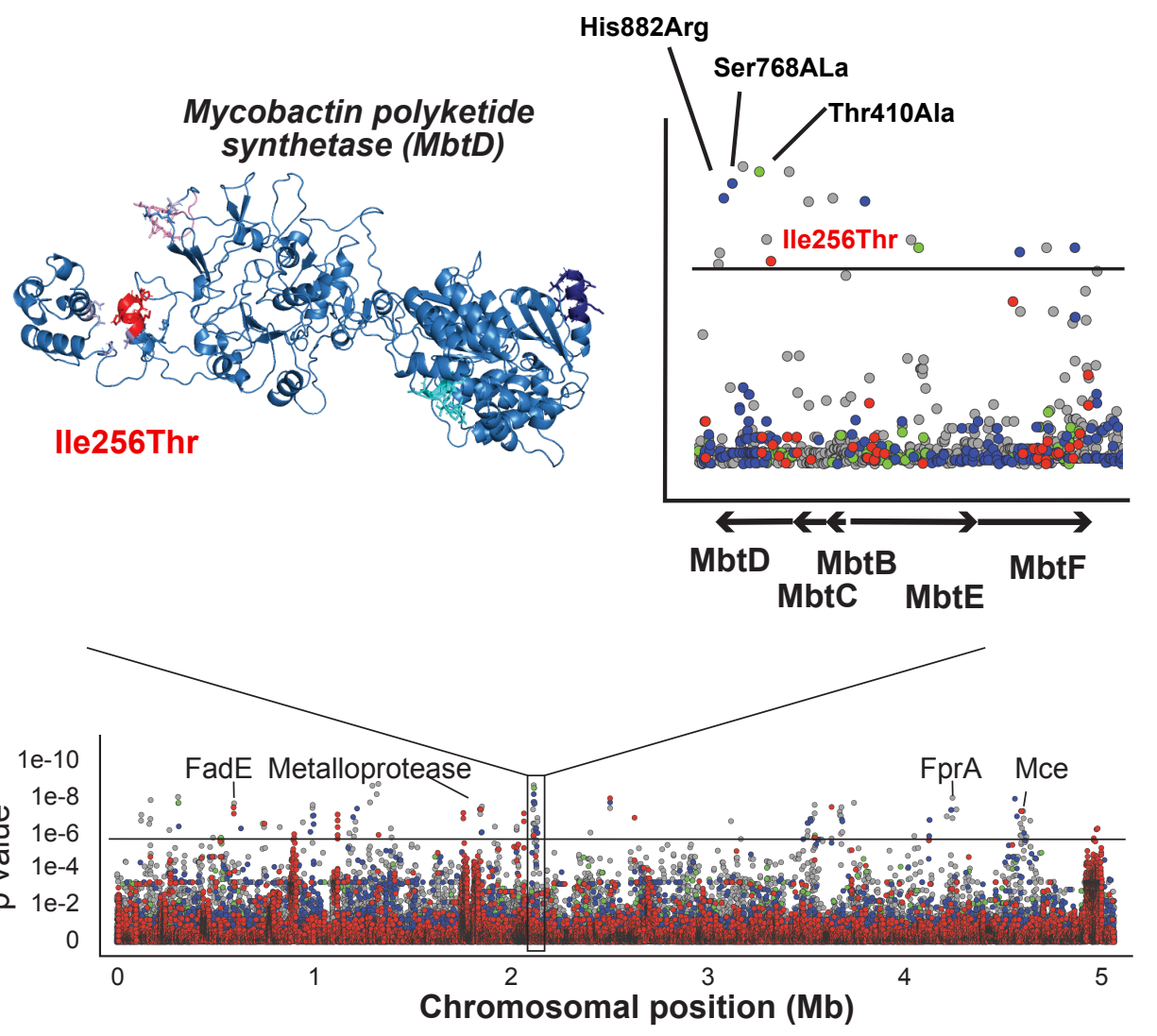


a.

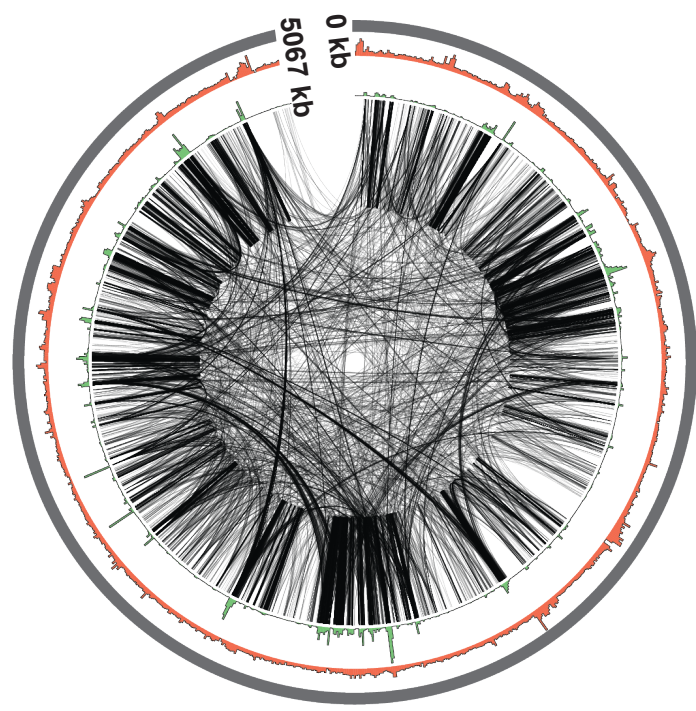

b.

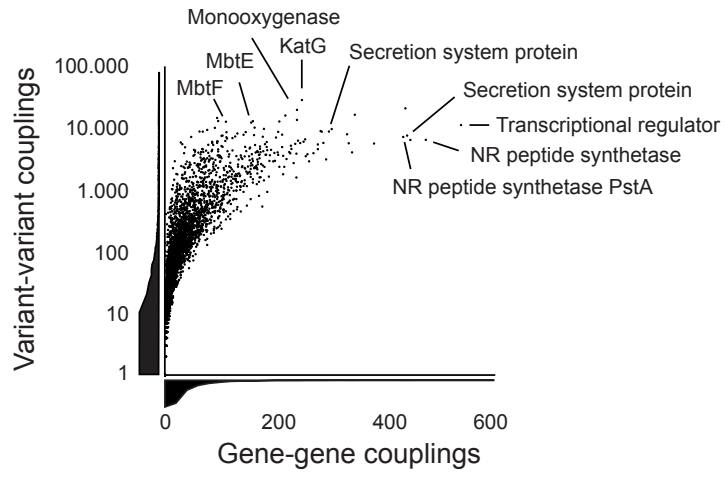

d.

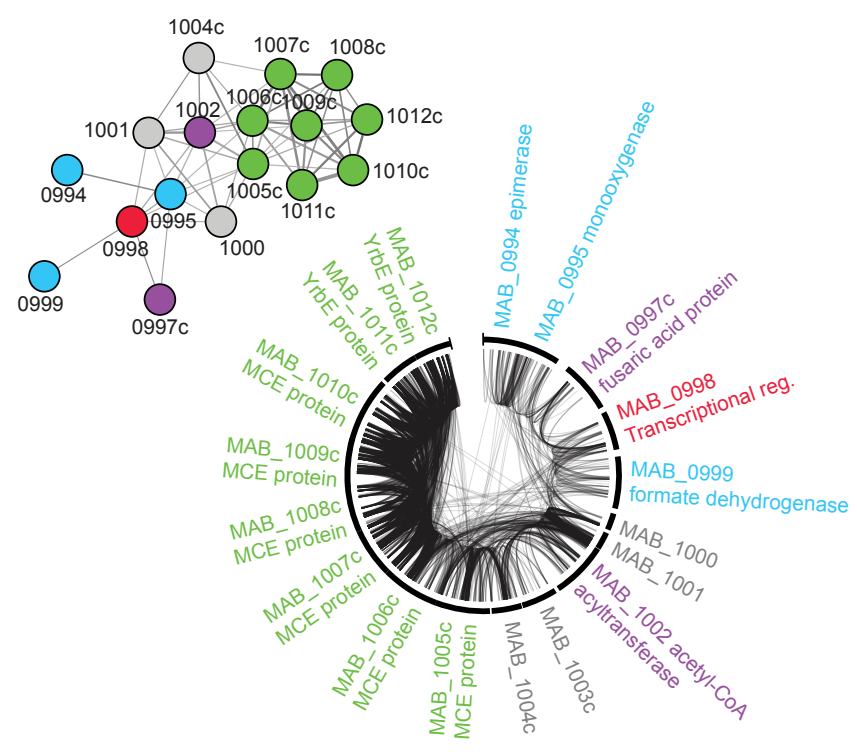

c.
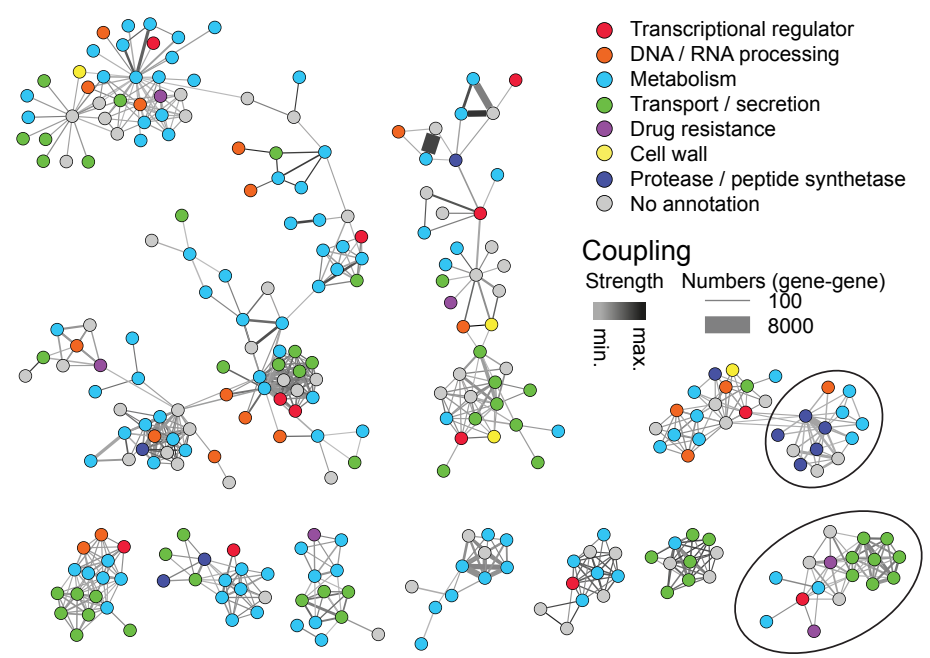

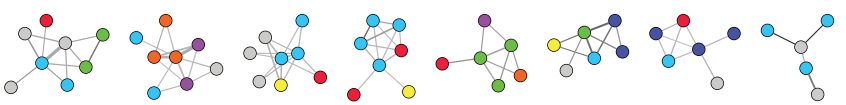

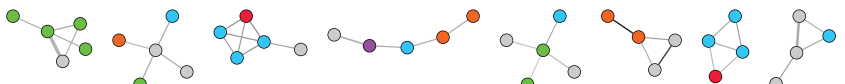

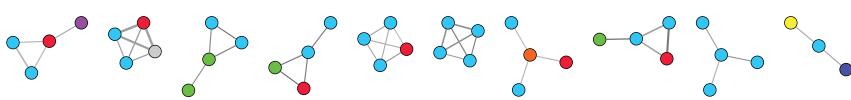

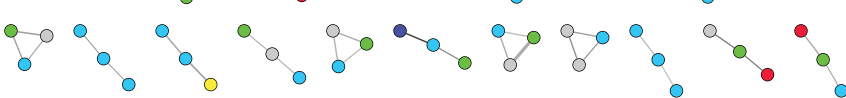

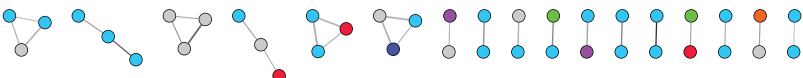

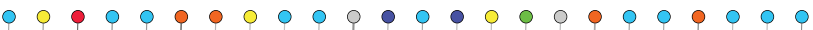

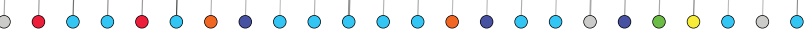

e.

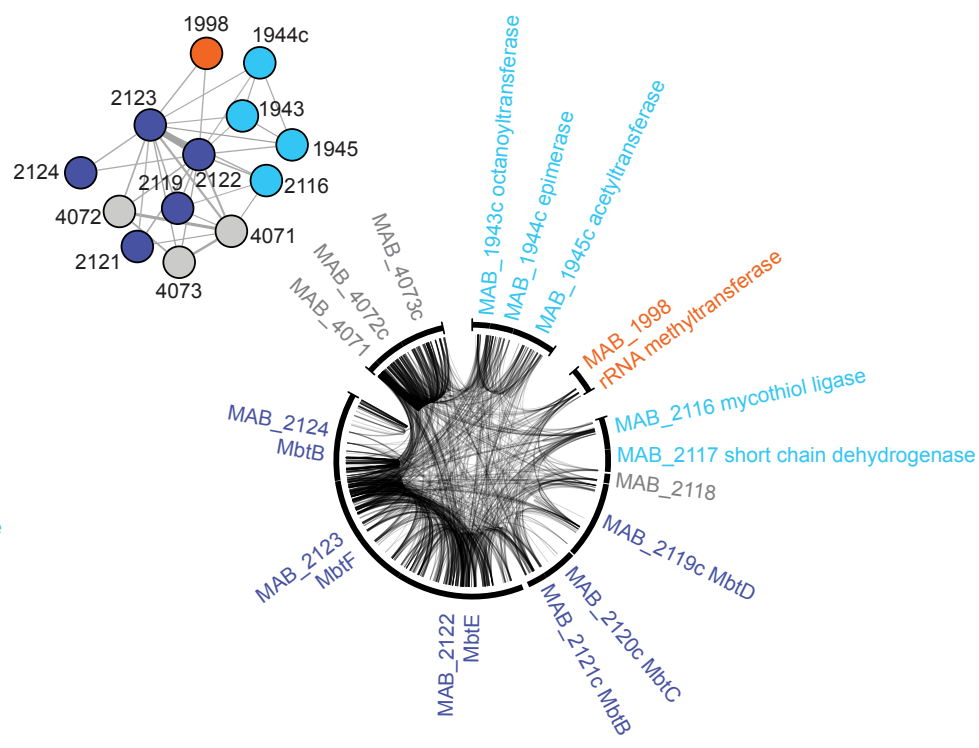


bioRxiv preprint doi: https://doi.org/10.1101/2021.10.18.464689; this version posted October 18, 2021. The copyright holder for this Figure afeprint (which was not certified by peer review) is the author/funder, who has granted bioRxiv a license to display the preprint in perpetuity. It is made available under aCC-BY 4.0 International license.

a.

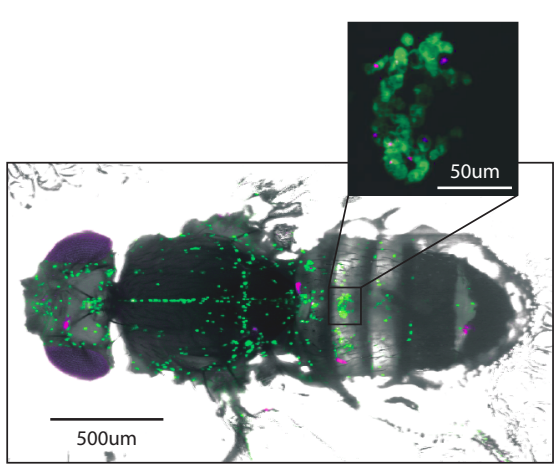

C.

Mean Drosophila survival Secretion system protein

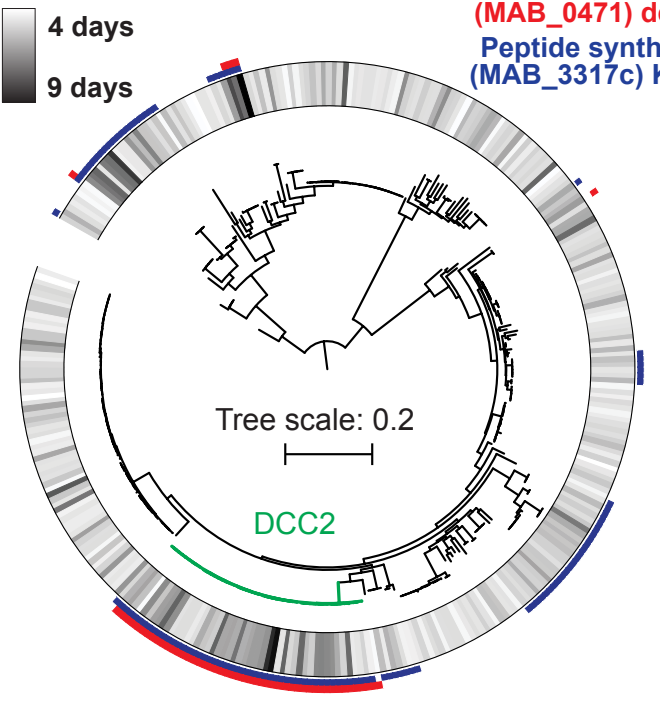

e.

- Empty vector control - MAB_3317c knockdown

- MAB_0471 knockdown - YidC knockdown
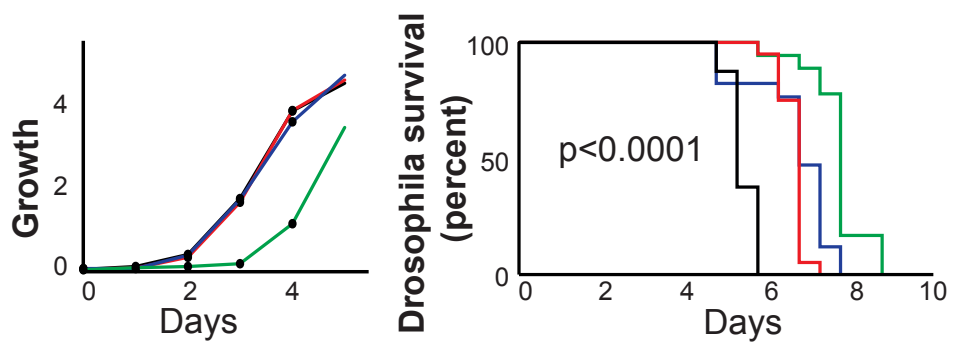

Drosophila survival

Secretion sytem protein

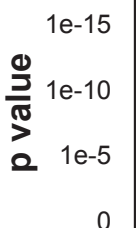

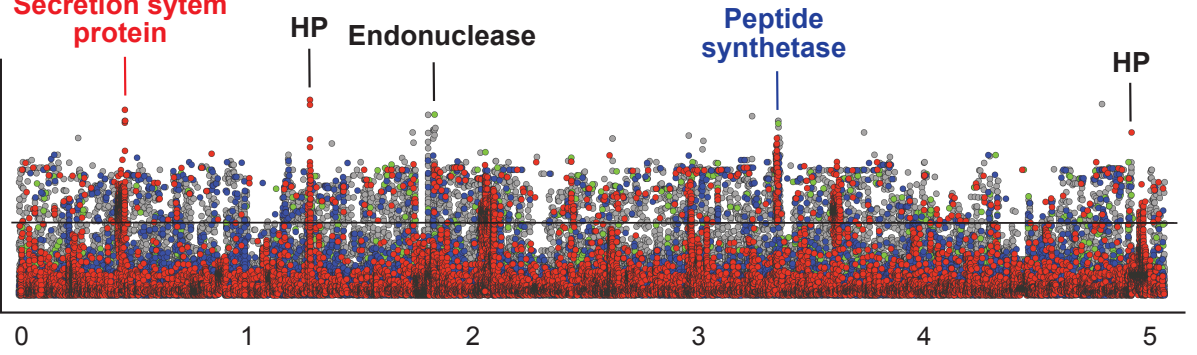

d.

Chromosomal position (Mb)

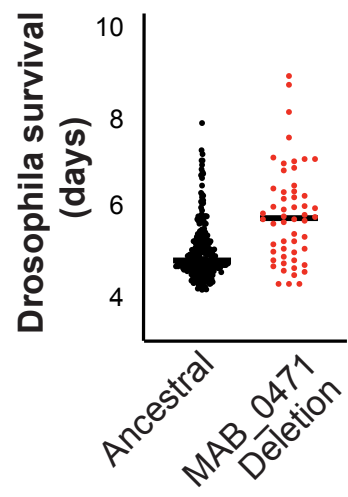

Ancestral MAB 0471

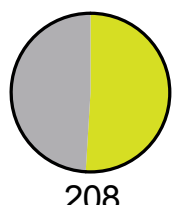

Deletion

f.
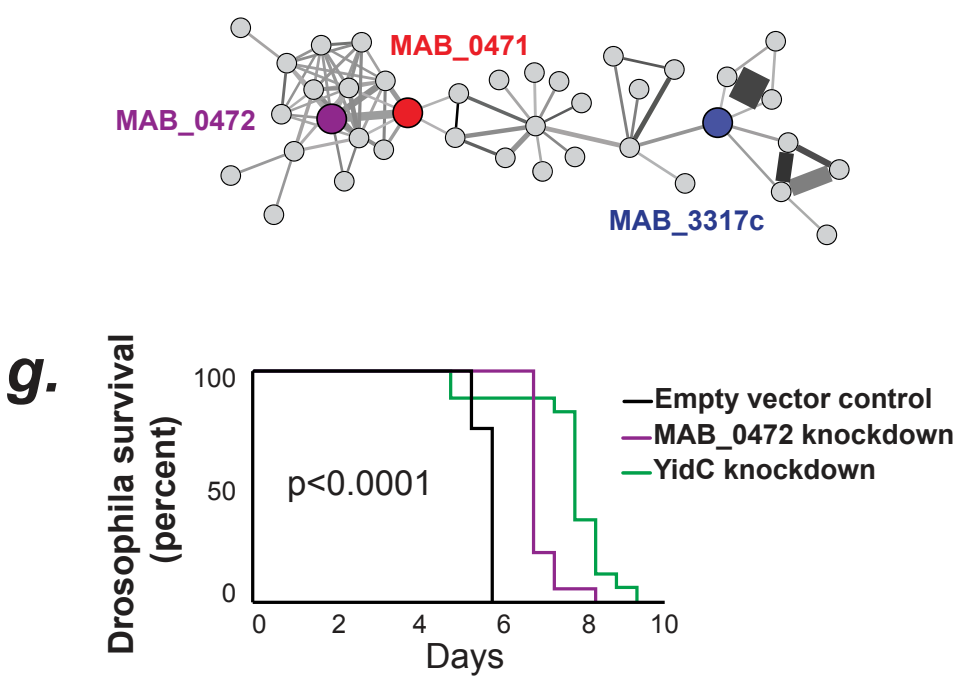